\title{
Utility of Optical Density of Picrosirius Red Birefringence for Analysis of Cross-Linked Collagen in Remodeling of the Peripartum Cervix for Parturition
}

\author{
Michael A Kirby ${ }^{1,2,3, *}$, Anne C Heuerman BS ${ }^{1}$ and Steven M Yellon ${ }^{1,3,4}$ \\ ${ }^{1 *}$ Lawrence D. Longo Center for Perinatal Biology, Loma Linda University School of Medicine, Loma Linda University. Loma Linda, California, USA \\ ${ }^{2}$ Departments of Pathology and Human Anatomy, Loma Linda University School of Medicine, Loma Linda University. Loma Linda, California, USA \\ 3Pediatrics, Loma Linda University School of Medicine, Loma Linda University. Loma Linda, California, USA \\ ${ }^{4}$ Basic Sciences, Division of Physiology, Loma Linda University School of Medicine, Loma Linda University. Loma Linda, California, USA
}

${ }^{\star}$ Correspondence to: Michael A. Kirby, Ph.D., Professor, Longo Center for Perinatal Biology, MRW A572, Loma Linda University School of Medicine. Loma Linda, California, 92350, USA; Email: mkirby@llu.edu; Tel: (909)558-4325

Received: June 15, 2018; Accepted: June 25, 2018; Published: June 28, 2018;

\begin{abstract}
We report on development of a rapid, quantitative analysis technique of collagen fibers in cross-linked structures to assess remodeling of the cervix during the transition from soft to ripening in preparation for birth. Optical density analysis of picrosirius red stain tissue using circular polarized birefringence light from fixed paraffin-embedded or frozen cervix from pregnant mice during phases of remodeling prior to birth. Data were analyzed using NIH Image J and extended recently to include studies of prepartum cervix in peripartum women. Our results, developed a rapid, consistent, technique to quantify cervical organization. This approach assesses the structure of collagen organization (the principle component of the cervix) and is essential for analysis of experimental outcomes that disrupt cervical morphology in rodent models of preterm birth. The technique, in this report has, for the first time permitted rapid, accurate assessment of the stages that define cervical ripening with large numbers of slides from individual animals. The approach integrates analysis of collagen organization, with distensability and inflammation, processes associated with cervical change before birth. This analysis further holds promise to evaluate other tissues, but also fibrolytic and fibrogenic changes in collagen associated with physiological or pathophysiological conditions.
\end{abstract}

Key words: pregnancy, remodeling, ripening, inflammation

\section{Introduction}

Identification of large protein using various stains have been used as early as the $17^{\text {th }}$ century to label extracellular, cellular, and sub-cellular organ structures in tissue [1]. More recently, improved fixation and specificity of stains has allowed quantification of small cellular components and their structural organization. Collagens are the most abundant proteins in humans with type 1 the majority (98\%) of the 28 identified types [2,3], and common in all vertebrate species. The human cervix, composed predominately of collagen fibers, serves as a barrier to the vaginal biome and protects the developing contents of the uterus during pregnancy [4]. Junqueira's group was the first to use picrosirius red stain to identify a reduction in collagen in cervix from intrapartum compared to nonpregnant women. Picrosirius red stains the principal forms of collagen in the cervix, mostly type 1 , though type 3 is present to a lesser extent [5]. As Lattouf more recently concluded, picrosirius red stain is "simple, sensitive and specific for collagen staining....particularly useful to reveal the molecular order, organization and/or heterogeneity of collagen fiber orientation in different connective tissues" [6].
Evidence supporting this conclusion led our lab, well over a decade ago, to develop a protocol that uses birefringence of circular polarized light from picrosirius red stained cervix sections to study the progression of remodeling during the progression from phases of softening to ripening [7-11]. In multiple strains of mice and rats, and more recently in women at term and preterm delivery, this technique is reliable, consistent and essential to assess degradation of collagen organization during late term normal pregnancy or experimental manipulations.

Given the utility of picrosirius red stain with birefringence, physiological remodeling and inflammation-induced premature ripening of the cervix, the goal of this report was to document the current state of our method, which is likely to have broad value to accurately study collagen of other tissues and assess pathological or healing of fibrotic processes.

\section{Methods}

Cervix from pregnant mice were processed by immersion fixation in $4 \%$ paraformaldehyde, paraffin embedded, sectioned at 
$10 \mu \mathrm{m}$, heated at $60^{\circ} \mathrm{C}$ for 45 minutes using a slide warmer, then subjected to xylene incubations to remove paraffin, and rehydrated through a graded series of ethanol. Sections were counterstained with hematoxylin to identify cell nuclei (for cell counting), and washed in distilled water to remove background stain. These tissue processing procedures have been previously detailed [7]. Collagen in cervix sections was stained using a picrosirius red kit (Polysciences Kit \#24901-500, Warrington, PA, USA). Following instructions, slides were first placed into Solution-A (a phosphomolybdic acid hydrate solution from the kit) for two minutes and then into Solution-B (Picrosirius Red-F3BA) for 60 minutes. Variations in incubation times of $\pm 20 \mathrm{~min}$ were not found to improve staining. Slides were placed into Solution-C (0.01 N HCL) for 2 minutes, dehydrated through an ascending series of ethanol, and placed in xylene before coverslipped with Permount (Fisher Scientific, \#SP15-100).

For analysis of collagen structural organization during phases of cervix remodeling, a Zeiss Axio Imager A1 microscope with circular polarized light filters was used to evaluate sections at 250x. In early development of this technique an additional microscope (Nikon Optiphot, with Plan apochromat objectives and Nomarski optics) was similarly validated for this method. In both cases, a Spot Pursuit 4MP digital camera was used for micrographs (Diagnostic Instruments, Sterling Heights, MI). In dim room illumination, initial regions of interest were visually identified. A representative sample of 4-6 grey scale photomicrographs were taken from 2 sections/mouse (8-12 total). A similar process was used for human tissue. Care was taken to avoid distorted morphology, including blood vessels, epithelial lumen, and section artifacts, i.e. tissue folds or tears. To ensure consistency in analysis across sections, as well as individuals and groups, microscope properties (field aperture, condenser, light intensity) were optimized and unchanged across viewing each study session. Cells counts were verified using two to three independent observers.

For image analyses, grey scale photomicrographs were filed into a date-labeled folder and batch processed to evaluate optical density using Image J software (NIH, Bethesda, MD; macro plugin attached in Appendix). At the outset, a global calibration macro was set with the first photomicrograph. The macro first digitized the photomicrograph into an 8-bit grayscale image (Figure. 1B). The program automatically and without bias centered a 9 -square box cross region for analysis. An average optical density (OD) was calculated with Rodbard transformation (performed by the program) for each of the 9 boxed regions together with total OD then exported as an output file to a Excel worksheet for statistical analyses. Data were analyzed by ANOVA ( $\mathrm{p}<0.05$ level of significance) with Tukey's post-hoc analysis following Levene's test (GraphPad Prism Software, Inc., La Jolla, CA).

\section{Results and Conclusions}

In the cervix from a nonpregnant mouse, collagen fibers were observed as a dense, uniform fascicle, stained by picrosirius red collagen fibers (Figure. 1A). The birefringence values further quantified this initial impression with values indicative of aligned and organized cross-linked fibers and bundles/fascicles (i.e., low light transmission values) characteristic of an unremodeled cervix. In contrast, by day 18 postbreeding (gestation term is 19 days, (Figure 1B), birefringence values were significantly altered, correlating with the presence of numerous gaps and disorganized, nonparallel collagen fibers. This morphology is characteristic of cervical ripening and consistent with early electron microscope studies $[12,13]$, as well as, consistent with ripening processes of hypertrophy, edema, and increased distensability of the cervix near term. Digitizing this image in grayscale combined with Rodbard transformation (performed by Image J), increased the sensitivity to discriminate the decline in picrosirius red-stained crosslinked collagen was enhanced (Figure 1C).

The utility of picrosirius red stain to understand structural remodeling of the cervix prior to parturition is illustrated by studies of two genetically modified (knockout, $\mathrm{KO}$ ) mouse models (Figure 2). In mice lacking the progesterone receptor $\mathrm{B}$ isoform, picrosirius red stain identified reduced extracellular cross-linked collagen in a temporal pattern that was similar as ripening found in wild type controls before term [14]. Moreover, in a second modified strain of mice lacking the prostaglandin F2 a receptor, pregnancy progressed while collagen structure was reduced similar to wild-type pregnant controls, however, neither labor nor term birth occurred in these animals [11]. The softening and ripening phases of cervix remodelling occurred in similar phases for both knockout models. Moreover, the use of our picrosirius red protocol critically distinguished, for the first time, the phases of cervical ripening from labor to birth in genetic modified animals in which progesterone mechanisms, thought to be essential for normal delivery, were deleted. In both models, the ability to assess cervical phases and collagen organization in processes thought as essential for normal inflammatory processes for parturition, were rapidly, quantitatively, easily, and determined.

These findings demonstrate the value of a rapid and replicable technique in which assessment of picrosirius red birefringence light can benefit analysis of critical biological questions about crosslinked collagen organization in tissue undergoing physiological change. Although used in the cervix, this technique has potential applications in other tissues or pathologies where fiber organization is disrupted. For example, this analysis may be useful to track the progression of fibrotic diseases of lung, kidney, or liver, as well as, to quantify therapeutic interventions of relevance for diverse pathologies including atherosclerosis, tumor growth, and metastatic processes, e.g. cardiovascular, scleroderma [7, 15]. Additionally, the use of transmitted light to determine collagen organization has been usefully employed in a variety of tissues, including human optic nerve [16], biological cells [17], and human skin [18].

Beyond tissue histology or biology, industrial applications would similarly benefit from a rapid assessment of other fibrous structures such as fabrics including Kevlar [19, 20], colloidal organization in liquid suspensions where knowledge of density, quantitative measurement, or dispersion properties are required.

Although useful in many biological situations, limitations of the use of picrosirius red stain to quantify collagen include its use in aqueous permeable fixed material and relatively thick ( 10um) sections $[20,21]$. Additionally, oral mucous and oral cavity fibrosis [22, 23], luminal crypts [21], or understandably, nonhomogeneous tissues with differing sub-layers encountered within the same region are problematic for analysis with this technique. 
A
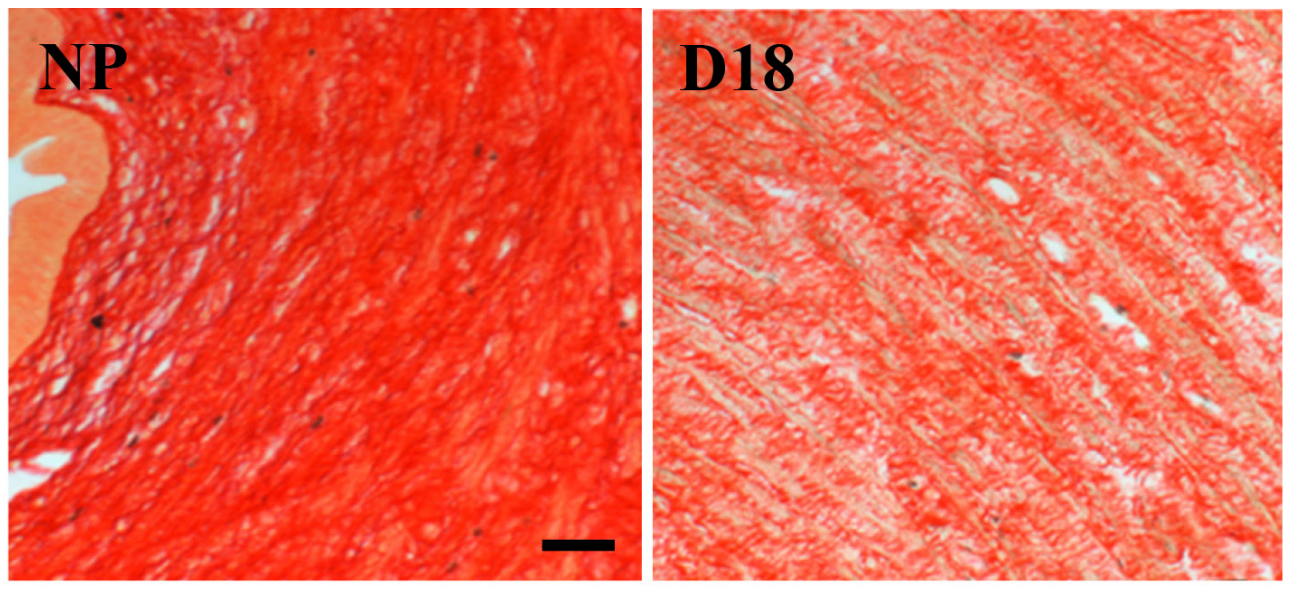

B
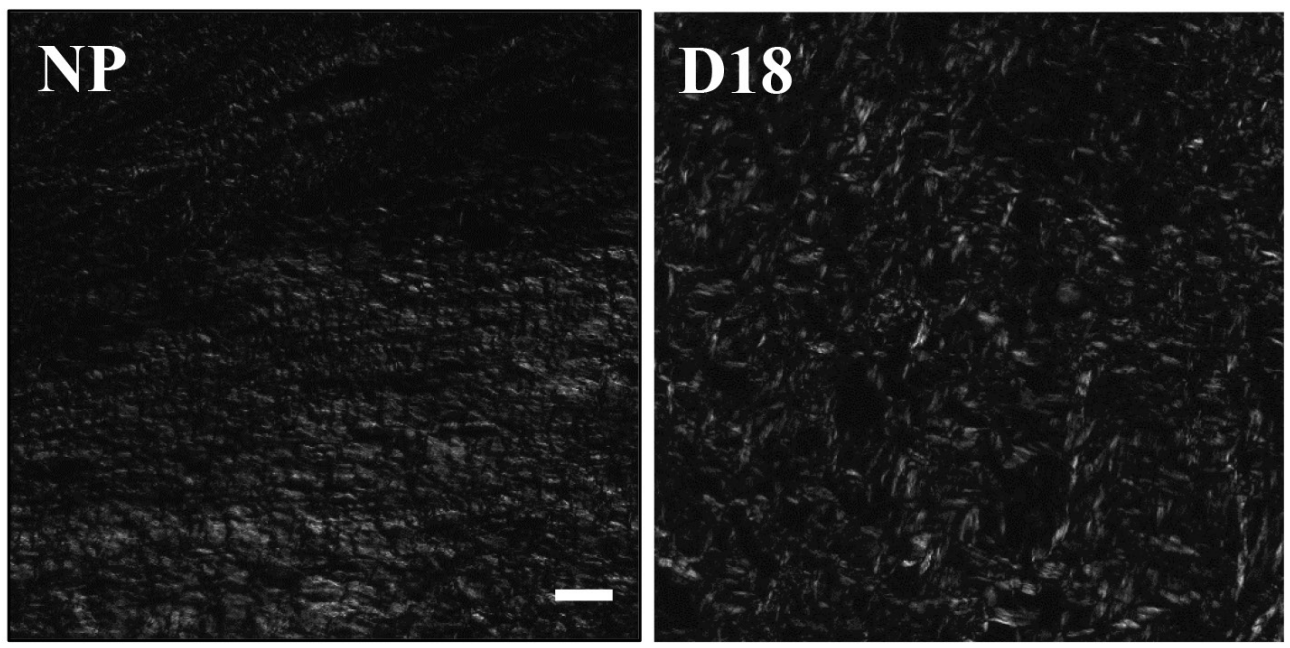

C
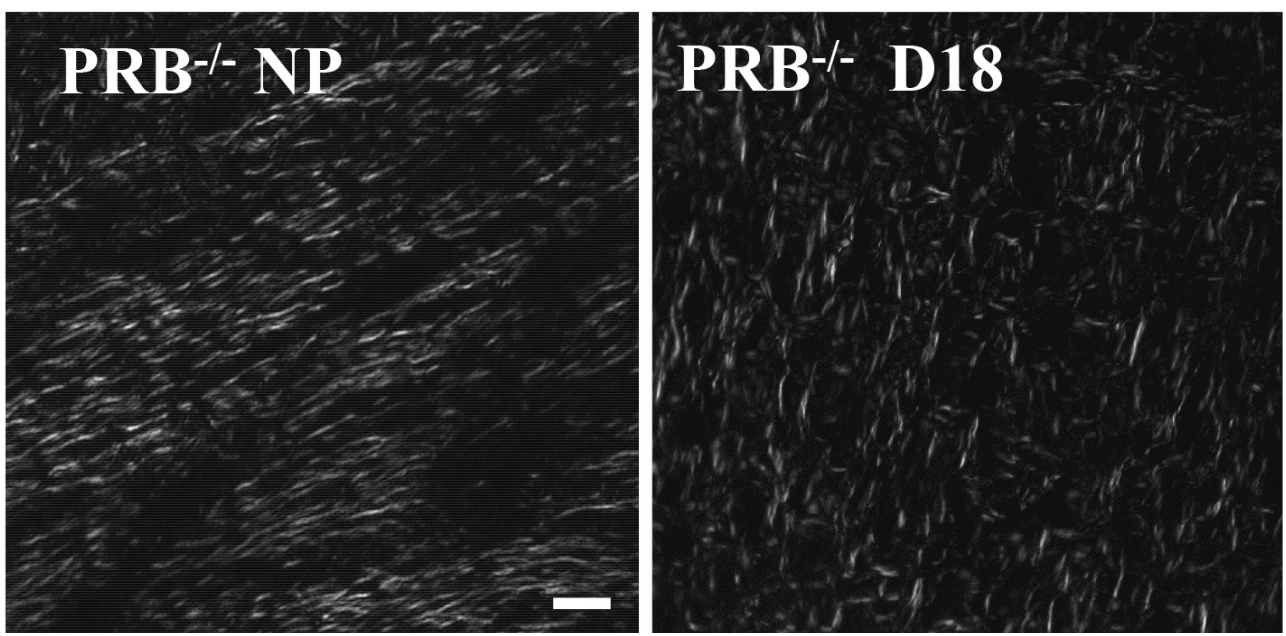

Figure 1A. Illustrative photomicrographs of Picrosirius Red stained, $10 \mu \mathrm{m}$ thick paraffin sections from the cervix of a non-pregnant (NP) and a day18 (d18) pregnant mouse cervix. Term gestation is 19-20 days. Note the ability to visualize individual collagen fibers and fascicles in both sections at this relatively low magnification ( $250 \mathrm{X}$, using a 20 power objective). The NP section is substantially darker reflecting the denser collagen fiber organization and corresponding reduced light transmission of cross-polarized light through the tissue. In contrast, at d18, as term gestation approaches, the collagen fiber organization is less dense and less organized. Individual fascicles have increased spacing, allowing greater light transmission through the tissue. Within Individual fascicles there is less organization and numerous irregularities as denoted by the overall lighter appearance (increased transmitted light and increased disorganization of individual fibers. Scale bar $=50 \mu \mathrm{m}$.

Figure 1 B and C. Photomicrographs of from nonpregnant (NP) and late term (d18) cervix from wild-type (WT) and progesterone receptor B knockout mice (PRBko). White areas illustrate birefringent illumination and more easily depicts differences in collagen organization. Fibers from non-pregnant animals have more linear structure, in contrast to late term D18 fibers which are dispersed and lack defined structure. Scale bar is $50 \mu \mathrm{m}$ for the two color images and $50 \mu \mathrm{m}$ for all grey scale images and is the same for all grey scale images. 


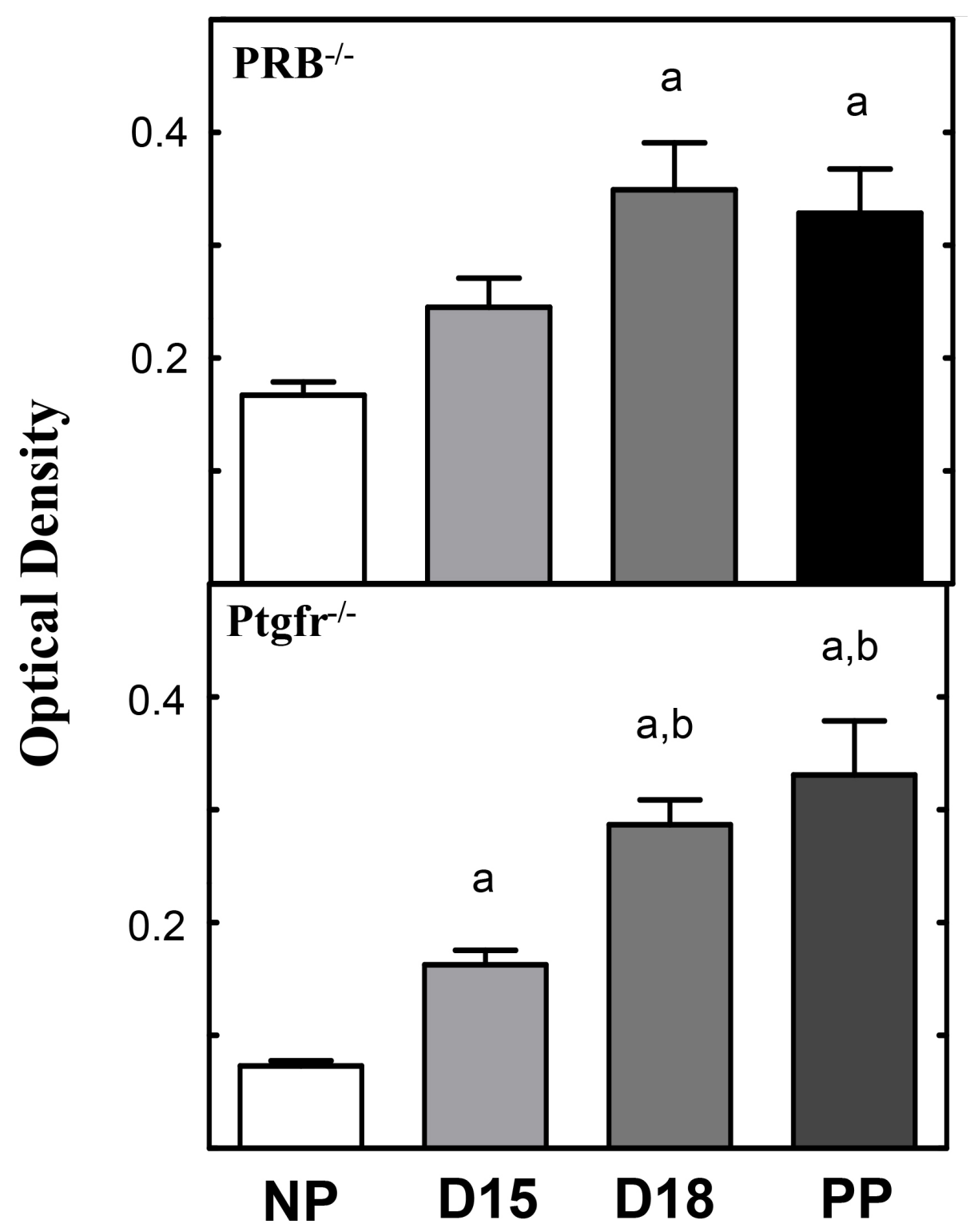

Figure 2. Histograms obtained from photomicrographs of Figure $1 \mathrm{~A}$ and B. Cervix optical density histograms (mean $\pm \mathrm{SEM})$ from pregnant Prostaglandin Receptor 2 knockout $\left(\mathrm{Ptgfr}^{-/}\right.$) and $\mathrm{PRB} /$ ) mice following picrosirius red processing (see methods). Optical density values denote an inverse relationship to collagen structure and organization. The more structured a tissue, the lower optical density values for that tissue. All statistical comparisons were performed using analysis of variance (ANOVA) with Tukey's post-hoc analysis. "a" equals comparison to non-pregnant, "b" denotes comparison to D15. [11, 14]

In summary, the technique we have developed combining picrosirius red stain and birefringence analysis using circular cross polarized light has evolved over several years into an easy, rapid and essential technique, for our continued analysis in experimental manipulations of the rodent, human, and potentially other nonhuman primate cervical tissue. This technique is essential for rapid, accurate analysis of several hundred sections we commonly employ for each cervix. Our experimental manipulations are pivotal for understanding cervical processes promoting normal term delivery in rodent experimental models with our ultimate goal to understand the central and sometimes subtle process in humans.

\section{Acknowledgements}

This work was supported, in part, by NIH grant \# R01-HD054931 (SY), and grateful support from the Department of Pediatrics, School of Medicine, Loma Linda University, and the John Mace Pediatric Research fund (MAK). All animal work was performed in accordance with an approved animal care protocol of the Loma Linda University Animal Care and Use Committee (IACUC), \#89002. 


\section{Competing Interests}

All authors declare and affirm the lack of competing interests, finical or otherwise, in this manuscript.

\section{Abbreviations}

ANOVA - analysis of variance

CA - State of California

$\mathrm{DIH}_{2} \mathrm{O}$ - double distilled water

DXX - estimated day post-conception

Inc. - incorporated business

MI - State of Michigan

NP - nonpregnant

OD - optical density

PP - postpartum, day of delivery animal

PRB ko - Progesterone receptor, gene deleted animal

Ptgfr ko - prostaglandin-F2-genetic gene-deleted animal

ROI - region of interest

WT - wild type

$250 \mathrm{X}$ - total optical magnification as viewed through microscope objective

\section{References}

1. Bracegirdle B (1977) The History of Histology: A Brief Survey of Sources. History of Science 15: 77-101.

2. Mescher AL, Junqueira LCU (2016) Junqueira's basic histology: Text and atlas (Fourteenth edition.). New York: McGraw-Hill Education.

3. Sabiston DC, Townsend CM (2012) Sabiston Textbook of Surgery: The Biological Basis of Modern Surgical Practice. Elsevier Saunders.

4. Junqueira LC, Zugaib M, Montes GS, Toledo OM, Krisztan RM, Shigihara KM (1980) Morphologic and histochemical evidence for the occurrence of collagenolysis and for the role of neutrophilic polymorphonuclear leukocytes during cervical dilation. Am J Obstet Gynecol. 138: 273-81.

5. Uldbjerg N, Ekman G, Malmstrom A, Olsson K, Ulmsten U (1983) Ripening of the human uterine cervix related to changes in collagen, glycosaminoglycans, and collagenolytic activity. Am J Obstet Gynecol 147: 662-6.

6. Lattouf R, Younes R, Lutomski D, Naaman N, Godeau G, et al. (2014) Picrosirius red staining: a useful tool to appraise collagen networks in normal and pathological tissues. J Histochem Cytochem 62: 751-758. [crossref]

7. Kirby MA, Heuerman AC, Custer M, et al. (2016) Progesterone Receptor-Mediated Actions Regulate Remodeling of the Cervix in Preparation for Preterm Parturition. Reprod Sci 23: 1473-83.

8. Dubicke A, Ekman-Ordeberg G, Mazurek P, Miller L, Yellon SM (2016) Density of Stromal Cells and Macrophages Associated With Collagen Remodeling in the Human Cervix in Preterm and Term Birth. Reprod Sci 23: 595-603.

9. Dobyns AE, Goyal R, Carpenter LG, Freeman TC, Longo LD, et al. (2015) Macrophage gene expression associated with remodeling of the prepartum rat cervix: microarray and pathway analyses. PLoS One 10: e0119782. [crossref]

10. Yellon SM, Burns AE, See JL, Lechuga TJ, Kirby MA (2009) Progesterone withdrawal promotes remodeling processes in the nonpregnant mouse cervix. Biol Reprod 81: 1-6.

11. Yellon SM, Ebner CA, Sugimoto Y (2008) Parturition and recruitment of macrophages in cervix of mice lacking the prostaglandin F receptor. Biol Reprod 78: 438-444. [crossref]

12. Feltovich H, Ji H, Janowski JW, Delance NC, Moran CC, Chien EK (2005) Effects of selective and nonselective PGE2 receptor agonists on cervical tensile strength and collagen organization and microstructure in the pregnant rat at term. Am J Obstet Gynecol 192: 753-60.

13. Clark K, Ji H, Feltovich H, Janowski J, Carroll C, Chien EK (2006) Mifepristoneinduced cervical ripening: structural, biomechanical, and molecular events. Am J Obstet Gynecol. 194: 1391-8.
14. Yellon SM, Oshiro BT, Chhaya TY, Lechuga TJ, Dias RM, et al. (2011) Remodeling of the cervix and parturition in mice lacking the progesterone receptor B isoform. Biol Reprod 85: 498-502. [crossref]

15. Kirby LS, Kirby MA, Warren JW, Tran LT, Yellon SM (2005) Increased innervation and ripening of the prepartum murine cervix. J Soc Gynecol Investig 12: 578-585. [crossref]

16. Cense B, Chen TC, Park BH, Pierce MC, de Boer JF (2002) Invivo depth-resolved birefringence measurements of the human retinal nerve fiber layer by polarizationsensitive optical coherence tomography. Optics letters 27: 1610-2.

17. Mourant JR, Freyer JP, Hielscher AH, Eick AA, Shen D, Johnson TM (1998) Mechanisms of light scattering from biological cells relevant to noninvasive optical-tissue diagnostics. Applied optics. 37: 3586-93.

18. Pierce MC, Strasswimmer J, Park BH, Cense B, de Boer JF (2004) Advances in optical coherence tomography imaging for dermatology. The Journal of investigative dermatology 123: 458-63.

19. Penn L, Larsen F (1979) Physicochemical properties of kevlar 49 fiber. Journal of Applied Polymer Science 23: 59-73.

20. Rich L, Whittaker P (2005) Collagen and Picrosirius Red Staining: A Polarized Light Assessment of Fibrillar Hue and Spatial Distribution Braz. J Morphol Sci 22: $97-104$.

21. Nazac A, Bancelin S, Teig B, et al. (2015) Optimization of Picrosirius red staining protocol to determine collagen fiber orientations in vaginal and uterine cervical tissues by Mueller polarized microscopy. Microscopy research and technique 78: $723-30$

22. Marcos-Garces V, Harvat M, Molina Aguilar P, Ferrandez Izquierdo A, Ruiz-Sauri A (2017) Comparative measurement of collagen bundle orientation by Fourier analysis and semiquantitative evaluation: reliability and agreement in Masson's trichrome, Picrosirius red and confocal microscopy techniques. J Microsc 267: $130-42$.

23. Kamath VV, Satelur K, Komali Y (2013) Biochemical markers in oral submucous fibrosis: A review and update. Dent Res J (Isfahan) 10: 576-584. [crossref]

\section{Citation:}

Michael A Kirby, Anne C Heuerman BS and Steven M Yellon (2018) Utility of Optical Density of Picrosirius Red Birefringence for Analysis of Cross-Linked Collagen in Remodeling of the Peripartum Cervix for Parturition. Integr Gyn Obstet $J$ Volume 1(2): 1-5. 